\title{
Area-minimizing Surfaces in Cones
}

\author{
Frank MORGAN
}

We show that a $k$-dimensional area-minimizing surface can pass through an acute conical singularity if and only if $k \geq 3$. The larger $k$, the more acute the conical singularity can be.

\section{Introduction.}

We consider $k$-dimensional area-minimizing surfaces in $\mathbf{R}^{n}$ with metric in polar coordinates

$$
d s^{2}=d r^{2}+\delta^{2} r^{2} d \Theta^{2} \quad(0<\delta<1)
$$

where $d \Theta$ is the standard metric on the unit sphere $\mathbf{S}^{n-1}$. This ambient space $M_{0}$ is isometric to the cone

$$
\left\{y=\delta^{-1}\left(1-\delta^{2}\right)^{\frac{1}{2}}|x|\right\}
$$

in Euclidean $(n+1)$-space.

It is easy to show (see [IT, Chapt. 9, Cor. 1.1], [CFG]) that a lengthminimizing curve avoids the vertex. We show (Corollary 3.2) that a twodimensional area-minimizing surface avoids the vertex. For $k \geq 3, k$ dimensional area-minimizing surfaces can in general pass through the vertex.

1.1 Theorem (see Theorem 3.1). A $k$-dimensional plane through the vertex is area-minimizing if and only if

$$
k \geq 3 \quad \text { and } \quad \delta^{2} \geq \frac{4(k-1)}{k^{2}} .
$$

One can compute after Simons [Sns] that this is the precise condition for stability as well (see Remark 3.1(6)). Our proofs after Lawlor [L] include a short proof of this stability result too. Every nonplanar regular stationary cone is unstable for $\delta^{2}<8(k-1) / k^{2}$. One may consider $\delta \geq 1$ as well as $0<\delta<1$. Of course the collection of stationary cones is the same for all $\delta$, and the larger $\delta$, the more likely a cone is to be stable or area-minimizing.

Every regular stationary cone is area minimizing for $\delta$ sufficiently large (Remark 3.1(4)). For example, Proposition 3.3 proves that the cone over 
$\mathbf{S}^{m} \times \mathbf{S}^{m}$ in $\mathbf{R}^{2 m+2}(m \geq 1)$ is area minimizing if and only if $\delta^{2} \geq 16 m /(2 m+$ $1)^{2}$. Our approach provides a convenient alternative definition of "strictly minimizing" for a cone in Euclidean space as "minimizing for some $\delta<1$ " (Section 3.6).

These results have applications to more general ambients with conical singularities (see Corollary 3.2).

Theorem 3.7 considers the product $M$ of a Euclidean line or $m$-space with the cone $M_{0}(1)$, which has a line or $m$-plane of conical singularities. A twodimensional area-minimizing cone at a singular point in $M$, for example, must be a sum of "horizontal" planes contained in the singular set and possibly (when $\operatorname{dim} M_{0}=2$ ) a single "vertical" plane equal to $M_{0}$.

1.2 Proofs. The proof of Theorems 1.1 and 3.1 adapts the methods of Lawlor [L] for deciding whether a cone in Euclidean space is area minimizing. Lawlor proves cones minimizing by constructing an area-nonincreasing retraction onto the cone, equivalent to a calibration. For an isoparametric hypercone (over a manifold with constant curvature), if the retraction does not exist in any wedge about the cone, then the cone is not stable. The existence of the retraction is equivalent to the existence of a solution to a certain initial value problem. In general this problem can be analyzed numerically. Fortunately in our cases it is amenable to rather easy exact analysis by hand, using test functions suggested by Lawson [Ls].

1.3 Acknowledgment. This work was inspired by the work of the 2000 Williams College NSF "SMALL" undergraduate research Geometry Group [CFG], consisting of Andrew Cotton, David Freeman, and John Spivack.

This work is partially supported by a National Science Foundation grant.

\section{Singular ambient, rectifiable currents, area minimization, tangent cones.}

In this chapter we consider the nature and regularity of area-minimizing surfaces in ambients $M_{0}$ with a conical singularity.

Let $M_{0}$ be $\mathbf{R}^{n}$ with metric $d s^{2}=d r^{2}+\delta^{2} r^{2} d \Theta^{2}(\delta>0)$, where $d \Theta$ is the standard metric on the unit sphere. Since this metric remains within a bounded factor of the Euclidean metric, the topology on the spaces of rectifiable and integral currents remains unchanged. Suppose that $\delta \leq 1$. Then $M_{0}$ is isometric to the cone $\left\{y=\frac{\sqrt{1-\delta^{2}}}{\delta}|x|\right\}$ in Euclidean $(n+1)$ space; hence area is lowersemicontinuous, and the Compactness Theorem $[\mathrm{M} 1,5.5]$ for compact Lipschitz neighborhood retracts, such as a large initial segment of $M_{0}$, guarantees the existence of an area-minimizing surface with a given boundary. 
Let $T$ be a $k$-dimensional area-minimizing rectifiable current through the origin of $M_{0}$. Inside $M_{0}$, the ratio of the area inside a ball $B(0, r)$ to $r^{k}$ is monotonically increasing function of $r$, by the same proof as in Euclidean space [M1, Thm. 9.3]. It follows that $T$ has an area-minimizing tangent cone at the origin (see [M1, Thm. 9.8]).

More generally consider an $n$-dimensional submanifold $M$ of Euclidean $\mathbf{R}^{N}$, with a conical singularity $P$, where $M$ is locally smoothly diffeomorphic to its tangent cone, which is a round spherical cone $M_{0}$ as previously considered. Let $T$ be a $k$-dimensional area-minimizing rectifiable current through $P$. Inside $M$, for distance $r$ from $p$ small, $e^{r}$ times the ratio of the area $f(r)$ inside an intrinsic ball $B(0, r)$ to $r^{k}$ is a monotonically increasing function of $r$, by a slight variation on the Euclidean proof [M1, Thm. 9.3]. (The estimate $f \leq \frac{r}{m} f^{\prime}$ is replaced by the estimate $\left(1-\frac{r}{m}\right) f \leq \frac{r}{m} f^{\prime}$, which holds because $M$ is well approximated by $M_{0}$.) It follows that $\mathrm{T}$ has an area-minimizing tangent cone at $p$ in $M_{0}$.

In all of these results, $M_{0}$ may be replaced by a product of Euclidean space with $M_{0}$.

\section{Minimal cones at conical singularities.}

Our main Theorem 3.1 characterizes area-minimizing and stable planes at a conical singularity. Theorem 3.7 considers lines and planes of conical singularities.

3.1 Theorem. In $\mathbf{R}^{n}$ with conical metric

$$
d s^{2}=d r^{2}+\delta^{2} r^{2} d \Theta^{2} \quad(0<\delta<1)
$$

a $k$-dimensional plane $P(1 \leq k \leq n-1)$ through the origin is areaminimizing if

$$
k \geq 3 \text { and } \delta^{2} \geq 4(k-1) / k^{2}
$$

and is not stable otherwise, i.e. if

$$
k<3 \text { or } \delta^{2}<4(k-1) / k^{2} .
$$

(4)Remark. Similarly each of Lawlor's numerous examples of areaminimizing cones in Euclidean space remains area-minimizing under our singular metric (1) for $\delta$ near 1 . This is because each satisfies Lawlor's criterion strictly ([L, p. 88]; see Sect. 3.6). For example, Proposition 3.3 proves that for $m \geq 3$ the cone over $\mathbf{S}^{m} \times \mathbf{S}^{m}$ remains area-minimizing if and 
only if $\delta^{2} \geq 16 m /(2 m+1)^{2}$. It is even true that the cone over $\mathbf{S}^{2} \times \mathbf{S}^{2}$ is areaminimizing if $\delta^{2} \geq 32 / 25>1$. Any regular minimal cone is area-minimizing for $\delta$ sufficiently large, as our extension of Lawlor's method shows.

(5)Remark. For $k \geq 2$, the asserted instability holds in the strongest sense: there is a variation fixing the origin with negative second variation of area. (For $k=1, P$ is not stationary for any $\delta<1$, though you have to move the origin to reduce length; cf. [IT, Chapt. 9, Cor. 1.1], [CFG]. For $k=2$, we mention that we have found variations moving the origin with second variation $-\infty$.) For $k \geq 3$ and $\delta^{2}=4(k-1) / k^{2}$, the planes $P$ of Theorem 3.1 give the first examples (beyond the 2-plane in Euclidean space) of what Lawlor [L, Thm. 3.3.4] calls "marginally stable" cones, which are, as he asserts, stable (indeed, in these cases, area minimizing; see also [L, 1.4 (7)]).

(6)Remark. Let $C$ be a hypercone over a submanifold $S$ of the unit sphere. Let $\alpha(p)^{2}$ denote the sum of the squares of the principal curvatures of $S$ in the standard metric. Our proof shows more generally that $C$ has nonnegative second variation under the conical ambient metric (1) if

$$
\delta^{2} \geq 4\left(k-1+\alpha^{2}\right) / k^{2}
$$

and negative second variation if

$$
\delta^{2}<4\left(k-1+\alpha^{2}\right) / k^{2},
$$

providing a necessary and sufficient condition for stability for $\alpha$ constant, as shown when $\delta=1$ by Lawlor [L, Cor. 4.4.6, Sect. 3.3] and earlier by Simons [Sns, Lemma 6.1.6], with a very short proof of sufficiency by Simon [Sn, Rmk. B.3]. Theorem 6.1.1 of Simons [Sns, mislabeled "Lemma" 6.1.1, p. 102] generalizes to show that a nonplanar regular minimal hypercone has negative second variation if

$$
\delta^{2}<8(k-1) / k^{2} .
$$

(Note that this is sharp for the cone over $\mathbf{S}^{m} \times \mathbf{S}^{m}$ of Proposition 3.3.) The only change in the proof is that in the computation of a certain eigenvalue $\lambda_{1}$, previously at most $-(k-1)$ (which Simons calls $-p$ ), there is a scaling by $\delta^{-2}$, and an additional contribution of $-\left(\delta^{-2}-1\right)(k-1)$ because the relevant Ricci curvature of our ambient is $r^{-2}\left(\delta^{-2}-1\right)$ [Pet, p.71]. (The shorter proof of [SSY, Sect. 3], [Sn, Appendix B], and [G, Chapt. 10] does not generalize immediately because the radial and tangential effects are not decoupled.) This result probably holds for singular minimal cones too. It 
would be nice to have a general proof that if a plane is not minimizing or stable then no cone is minimizing or stable.

Proof of Theorem 3.1. We may assume that $n=k+1$, because the map $P \times \mathbf{R}^{n-k} \rightarrow P \times \mathbf{R}$ which maps $(x, y)$ to $(x,|y|)$ is distance nonincreasing.

We adapt the methods of Lawlor [L] for deciding whether a cone in Euclidean space is area-minimizing. Lawlor proves cones minimizing by constructing an area-nonincreasing retraction onto the cone, equivalent to a calibration. If the retraction does not exist in any wedge about the cone, then the cone is not stable.

Lawlor [L, Chapter 3] shows that the existence of the retraction in Euclidean space is equivalent to the existence of a solution $g(t)$ to the initial value problem

$$
g^{2}+\left(-t g+\frac{1+t^{2}}{k} g^{\prime}\right)^{2} \leq\left(1+t^{2}\right) p(t)^{2}, g(0)=1,
$$

or equivalently the equality. The retraction is globally defined if $g$ hits 0 soon enough.

The function $p(t)$ is a polynomial depending on the curvature of the cone. For us, $p(t)=1$. For an isoparametric hypercone, $p(t)=1-\frac{1}{2} \alpha^{2} t^{2}+\ldots$, where $\alpha^{2}$ is the sum of the squares of the principal curvatures.

The variable $t$ represents the tangent $\tan \varphi$ of the angular distance from the cone; for planes $P$, we just need $g$ to hit 0 before $t=\infty$.

For our metric (1) the differential inequality takes the form

$$
g^{2}+\delta^{-2}\left(-t g+\frac{1+t^{2}}{k} g^{\prime}\right)^{2} \leq\left(1+t^{2}\right) p(t)^{2}, g(0)=1,
$$

with $p(t)=1$. The only change in the derivation occurs in the middle of page 27 of [L]. Replacing Cartesian coordinates $r, z$ by polar coordinates $\rho, \varphi$ transforms

to

$$
\left(g-\frac{\tan \varphi}{k} g^{\prime}\right) d r+\frac{g^{\prime}}{k} d z
$$

$$
\cos \varphi g d \rho+\left(-\sin \varphi g+\sec \varphi \frac{g^{\prime}}{k}\right) \rho d \varphi
$$

which has norm at most 1 in our singular metric precisely when (7) holds.

Our expression (8) suggests the convenient substitution $g=\cos \beta / \cos \varphi$, under which (7) becomes

$$
\left|\frac{d \beta}{d \varphi}+(k-1) \frac{\tan \varphi}{\tan \beta}\right| \leq \delta k, \quad \beta(0)=0 .
$$


If we take $\beta \geq 0$, the operative inequality is

$$
\frac{d \beta}{d \varphi} \leq \delta k-(k-1) \frac{\tan \varphi}{\tan \beta}, \quad \beta(0)=0 .
$$

More generally, without taking $p(t)=1$, the substitution $g=p \cos \beta / \cos \varphi$ yields

$$
\frac{d \beta}{d \varphi} \leq \delta k-(k-1) \frac{\tan \varphi}{\tan \beta}+q \frac{\sec ^{2} \varphi}{\tan \beta},
$$

where $q=p^{\prime}(t) / p(t)<0$. If $\delta$ is large, $\beta$ can hit $\pi / 2$ very fast, proving area minimization for any regular minimal cone as asserted in Remark (4).

First we assume that $k \geq 3$ and $\delta^{2} \geq 4(k-1) / k^{2}$ and prove $P$ area minimizing. Fortunately, instead of solving (7) numerically after Lawlor, we can guess an excellent solution after Lawson [Ls, p.245]

$$
\begin{aligned}
& \cos \beta=\cos ^{(1+\gamma)} \varphi, \quad(\gamma>0) \\
& d \beta / d \varphi=(1+\gamma) \tan \varphi / \tan \beta .
\end{aligned}
$$

Condition (9) becomes

$$
(1+\gamma / k) \tan \varphi \leq \delta \tan \beta .
$$

Squaring both sides and letting $z=\sec ^{2} \varphi \geq 1$, so that $\sec ^{2} \beta=z^{1+\gamma}$, yields

$$
(1+\gamma / k)^{2}(z-1) \leq \delta^{2}\left(z^{1+\gamma}-1\right) \quad(z \geq 1) .
$$

Since $\left(z^{1+\gamma}-1\right) /(z-1)$ is increasing, it suffices to check the inequality in the limit as $z \rightarrow 1$ :

$$
(1+\gamma / k)^{2} \leq \delta^{2}(1+\gamma)
$$

Plugging in the optimal $\gamma=\delta^{2} k^{2} / 2-k$ yields

$$
\delta^{2} \geq 4(k-1) / k^{2}
$$

as asserted. Note that this implies that $\gamma \geq k-2>0$ for $k \geq 3$. We conclude that $P$ is area minimizing if $k \geq 3$ and $\delta^{2} \geq 4(k-1) / k^{2}$.

Second we assume that $\delta^{2}<4(k-1) / k^{2}$ or $k<3$ and show that $P$ is not stable. As in [L, Sect. 4.3], a smaller competitor can be constructed in a wedge of angular radius $\varphi_{0} \leq \pi / 2$ about $P$ if there is a solution to

$$
\frac{d \beta}{d \varphi}=\delta k-(k-1) \frac{\tan \varphi}{\tan \beta}
$$


with $\beta(0)>0$ and $\beta\left(\varphi_{0}\right)=0$, as occurs for any small $\varphi_{0}$ if the Jacobian of the associated system

$$
\begin{gathered}
d \beta / d s=\delta k \tan \alpha-(k-1) \tan \varphi \\
d \varphi / d s=\tan \beta
\end{gathered}
$$

has imaginary eigenvalues, i.e. when the discriminant

$$
(\delta k)^{2}-4(k-1)<0
$$

as desired. When $k=2$, this inequality holds for all $0<\delta<1$. When $k=1$, this whole argument does not apply, but the case $k=1$ is trivial: a line $P$ through the vertex of a circular cone is not even stationary although, unlike the cases $k>1$, to do better you have to move it off the singular point.

The only difference in the construction from Lawlor [L, pp. 58-59] is that in our coordinates, $-\alpha$ is the angle that the competing integral curve makes with the radial direction.

To verify that $P$ has negative second variation, note that by Lawlor's construction [L, p. 59] the resulting smooth family of smaller competitors reduces area at least by a factor $g(0)=\cos \beta(0)$. Meanwhile $\beta(0)$, the angle the competitor makes with $P$ at their common boundary, is asymptotically proportional to the size of the variation (in any norm). Therefore the second variation of area is negative.

To obtain the instability condition for more general isoparametric hypercones asserted in Remark (6), we include the extra term of (10) involving $q=-\alpha^{2} t+\ldots$, so that the instability condition (12) becomes

$$
(\delta k)^{2}-4\left(k-1+\alpha^{2}\right)<0,
$$

as asserted in Remark (6).

3.2 Corollary. Let $M$ be an $n$-dimensional submanifold of $\mathbf{R}^{N}$ with a conical singularity $p$, where the tangent cone $M_{0}$ is $\mathbf{R}^{n}$ with metric $d s^{2}=$ $d r^{2}+\delta^{2} r^{2} d \Theta^{2}(0<\delta<1)$. If $k \leq 2$ or if $3 \leq k=n-1 \leq 7$ and $\delta^{2}<$ $4(k-1) / k^{2}$, then no $k$-dimensional area-minimizing hypersurface (rectifiable current) passes through the singularity (unless of course its given boundary does).

Remarks. The hypothesis $\delta^{2}<4(k-1) / k^{2}$ is sharp, even for $M=M_{0}$, since otherwise by Theorem 3.1, planes through $p$ are area minimizing.

The hypothesis $k \leq 7$ is probably unnecessary. One would have to eliminate for example a cone over a singular hypersurface in $\mathbf{S}^{8} \subset M_{0}$; here $\mathbf{S}^{8}$ has (a multiple of) the standard metric. 
Proof. To obtain a contradiction, suppose that there is such an areaminimizing hypersurface. A tangent cone $C$ at $p$ is area minimizing for the given metric on $\mathbf{R}^{n}$ and hence by comparison for the Euclidean metric too, since a competitor would be less radial and hence more tangential than $C$.

By standard regularity [M1, Chapter 8], $C$ must be a plane (possibly with multiplicity) or (only if $k=2$ and $n \geq 4$ ) a sum of planes or (only if $k=7)$ a regular minimal cone.

If $C$ is a plane, by Theorem $3.1, k \geq 3$ and $\delta^{2} \geq 4(k-1) / k^{2}$, the described contradiction. If $C$ is a nonplanar regular minimal cone, work of Simons (see Remark 3.1(6)) implies that $\delta^{2} \geq 8(k-1) / k^{2}$, a stronger contradiction.

3.3 Proposition. In $\mathbf{R}^{2(m+1)}(m \geq 1)$ with metric $d s^{2}=d r^{2}+$ $\delta^{2} r^{2} d \Theta^{2}(\delta>0)$, the cone $C$ over $\mathbf{S}^{m} \times \mathbf{S}^{m}$ is area minimizing if $\delta^{2} \geq$ $16 m /(2 m+1)^{2}$ and unstable if $\delta^{2}<16 m /(2 m+1)^{2}$.

Remarks. Theorem 3.1 and Proposition 3.3 are special cases: in general, minimal cones remain stable longer than area minimizing. Even in Euclidean space, the cone over $\mathbf{S}^{1} \times \mathbf{S}^{5}$ in $\mathbf{R}^{8}$ is stable but not area minimizing [Se], as verified by rigorous numerical analysis [L, Sect. 5.1]. The hand estimates of Lawson [Ls, Thm. 5] are not sharp enough even to prove that the cone over $\mathbf{S}^{2} \times \mathbf{S}^{4}$ is area minimizing in Euclidean $\mathbf{R}^{8}$.

Proposition 3.4 treats the easier special case of the cone over $\mathbf{S}^{0} \times \mathbf{S}^{0}$.

Proof. The proof is essentially the same as the proof of Theorem 3.1 after Lawlor. For $\mathbf{S}^{m} \times \mathbf{S}^{m}$, the sum of the squares of the principal curvatures $\alpha^{2}=2 m$ [L, Table 5.5.1]. By Remark 3.1(6), the cone $C$ has a negative second variation leaving the origin fixed if $\delta^{2}<16 m /(2 m+1)^{2}$. Conversely, to prove the cone $C$ area minimizing, it suffices to find a nonnegative solution of 3.1(10) with $\beta(0)=0$ which vanishes by $\pi / 4 \quad\left(\frac{1}{2} \cos ^{-1}(1-2 m / 2 m)\right.$ of [L, p. 68]). For this example, $p(t)=\left(1-t^{2}\right)^{m}[\mathrm{~L}$, Table 5.5.1] and hence $q(t)=p^{\prime}(t) / p(t)=-2 m t /\left(1-t^{2}\right)$. After Lawson we try

$$
\cos \beta=\cos ^{1+\gamma} 2 \varphi \quad(\gamma>0)
$$

with $t=\tan \varphi$. Condition 3.1(10) becomes

$$
(1+(2 \gamma+1) / k) \tan 2 \varphi \leq \delta \tan \beta
$$

with $k=2 m+1$. As before, this holds with $\gamma=\delta^{2} k^{2} / 8-k / 2-1 / 2$ if

$$
\delta^{2} \geq 8(k-1) / k^{2}=16 m /(2 m+1)^{2}
$$

as asserted. Note that

$$
\gamma=k / 2-3 / 2
$$


is positive if $m \geq 2(k \geq 5)$ while a limit argument with $\gamma$ increased by $\varepsilon$ is required for $m=1(k=3)$.

3.4 Proposition. In $\mathbf{R}^{2}$ with metric $d s^{2}=d r^{2}+\delta^{2} r^{2} d \Theta^{2}(\delta>0)$, the $X$ or cone over $\mathbf{S}^{0} \times \mathbf{S}^{0}$ is length minimizing if and only if $\delta \geq 2$, and it has nonnegative second variation if and only if $\delta \geq 1$.

Proof. The ambient manifold is just a cone with vertex angle $2 \pi \delta$. It is trivial that the $X$ has nonnegative second variation if and only if $\delta \geq 1$.

The $X$ is minimizing if and only if there is a solution to equality 3.1(9) such that $\beta$ hits $\pi / 2$ by the time $\varphi$ hits $\pi / 4$. In this case 3.1(9) becomes simply $d \beta / d \varphi=\delta$, so that $X$ is minimizing if and only if $\delta \geq 2$.

3.5 Remark. Of course, by comparison, results $3.1-3.4$ apply in $\mathbf{R}^{n}$ with metric of the form

$$
d s^{2}=d r^{2}+f(r, \theta)^{2} r^{2} d \Theta^{2} .
$$

3.6 Strict minimization. As a possibly convenient alternative to the definition of Hardt and Simon [HS, §3], one could define a cone $C$ in Euclidean space, the $\delta=1$ case of our metric

$$
d s^{2}=d r^{2}+\delta^{2} r^{2} d \Theta^{2}
$$

to be "strictly minimizing" if it remains minimizing for some $\delta<1$. From this definition it follows immediately that any smooth variation of compact support has strictly positive second variation of area. Indeed, any variation must somewhere turn radial toward tangential, introducing a second order effect in $\delta$. Since the second variation of area is nonnegative for some $\delta<1$, it must be strictly positive for $\delta=1$.

Such strict minimization holds whenever Lawlor's criterion holds strictly [L, Sect. 6.1], and conversely for regular isoparametric hypercones. Likewise the Hardt-Simon definition holds wherever Lawlor's criterion holds strictly ([L, Chapt. 7, (3)], [HS, Thm. 3.2(v)]). It is not known whether the HardtSimon definition implies even strict stability [HS, Rmk. 3.3(3)].

The Hardt-Simon definition for a $k$-dimensional cone says roughly that the extra cost of avoiding the $\varepsilon$-ball about its vertex is at least proportional to $\varepsilon^{k}$.

The following theorem considers for example ambients with a line of conical singularities.

3.7 Theorem. Consider $\mathbf{R}^{m} \times \mathbf{R}^{n}$ with metric

$$
d s^{2}=d s_{1}{ }^{2}+\left(d r^{2}+\delta^{2} r^{2} d \Theta^{2}\right) \quad(0<\delta<1, m \geq 0, n \geq 2)
$$


and a $k$-dimensional area-minimizing cone $C$ at the origin not contained in $\mathbf{R}^{m} \times\{0\}$.

For $k=1$, there is no such $C$.

For $k=2$, one must have $n=2$, and $C$ is $\{0\} \times \mathbf{R}^{2}$, possibly plus an area-minimizing sum of planes in $\mathbf{R}^{m} \times\{0\}$.

For $k \geq 3$, for $\delta$ near 1 , every plane of the form $P_{1} \times P_{2}$ with $\operatorname{dim} P_{2} \geq 3$ is area minimizing, and in general there are others, such as the cone over $\mathbf{S}^{3} \times \mathbf{S}^{3}$ in $\{0\} \times \mathbf{R}^{8}$.

Remark. The classification of area-minimizing cones for the metric (1) is open even for 3 -dimensional cones in $\mathbf{R}^{1} \times \mathbf{R}^{3}$ or $\mathbf{R}^{2} \times \mathbf{R}^{2}$. An areaminimizing 3-plane in $\mathbf{R}^{2} \times \mathbf{R}^{2}$ must have a factor in the first $\mathbf{R}^{2}$ and hence be of the form $\mathbf{R} \times \mathbf{R}^{2}$ by the $k=2$ case of Theorem 3.5. As far as I know, known examples of stationary cones in $\mathbf{R}^{m} \times \mathbf{R}^{n}$ with metric (1) are products $C_{1} \times C_{2}$ of stationary cones $C_{1} \subset \mathbf{R}^{m}, C_{2} \subset \mathbf{R}^{n}$.

Proof. For $k=1, C$ must be a line. $C$ lies in $C \times\left(\mathbf{R}^{n} \cap C^{\perp}\right)$, an $\mathbf{R}^{n}$ with metric $d s^{2}=d r^{2}+\delta(x)^{2} r^{2} d \Theta^{2}$ with $\delta_{0}=\max \delta(x)<1$. By Theorem $3.1, C$ is not minimizing in $\mathbf{R}^{n}$ with metric $d s^{2}=d r^{2}+\delta_{0}^{2} r^{2} d \Theta^{2}$. Since $C$ is entirely radial, $C$ is certainly not minimizing in $\mathbf{R}^{n}$ with metric $d s^{2}=$ $d r^{2}+\delta(x)^{2} r^{2} d \Theta^{2}$, the desired contradiction.

For $k=2, C$ is a cone over a system of simple closed geodesics in the unit sphere $S$ with coordinates $\left(\Theta_{1}, \Theta_{2}, \varphi\right), \Theta_{1} \in \mathbf{S}^{m-1}, \Theta_{2} \in \mathbf{S}^{n-1}, 0 \leq \varphi \leq \pi / 2$, typical point $\left((\cos \varphi) \Theta_{1},(\sin \varphi) \Theta_{2}\right)$, and metric $d s^{2}=d \varphi^{2}+\left(\cos ^{2} \varphi\right) d \Theta_{1}{ }^{2}+$ $\delta^{2}\left(\sin ^{2} \varphi\right) d \Theta_{2}{ }^{2}$. (One example to think of is the 2-sphere with metric $d s^{2}=$ $d \varphi^{2}+\delta^{2} \sin ^{2} \varphi d \Theta^{2}$.) Any geodesic $\gamma$ with $\varphi$ constant lies in $\mathbf{R}^{m} \times\{0\}$ or $\{0\} \times \mathbf{R}^{n}$. If $\gamma$ lies in $\mathbf{R}^{m} \times\{0\}$, the cone over it is a plane in $\mathbf{R}^{m} \times\{0\}$. If $\gamma$ lies in $\{0\} \times \mathbf{R}^{n}$, then the cone over $\gamma$ is area-minimizing if and only if $n=2$ by Theorem 3.1. If $\varphi$ is not constant, consider the point $p$ on $\gamma$ where $\varphi$ is smallest. If $\varphi(p)=0$, then $\gamma$ lies in $\mathbf{S}^{n} \subset\left(\mathbf{R}^{1} \times \mathbf{R}^{n}\right)$, which has an isolated singularity at $p$ of the type of Theorem 3.1, so the tangent cone $C_{1}$ to $\gamma$ at $p$ is not minimizing. Hence the tangent cone $\mathbf{R} \times C_{1}$ to the cone over $\gamma$ at $p$ is not minimizing and the cone over $\gamma$ is not minimizing, a contradiction. Therefore $\varphi(p)>0$. By comparison with the round sphere, we see that $\gamma$, extended in both directions from $p$, meets itself on the other side of $S$ at some angle less than 180 degrees, a contradiction. We conclude that $n=2$ and $C=\{0\} \times \mathbf{R}^{2}$, possibly plus an area-minimizing sum of planes in $\mathbf{R}^{m} \times\{0\}$ (characterized in [M2, Cor. 4] as all complex for some orthogonal complex structure on their span). Note that any such $C$ is area minimizing, because projection onto $\mathbf{R}^{m} \times\{0\}$ plus projection onto $\{0\} \times \mathbf{R}^{n}$ is area nonincreasing ([M3, Lemma 5.2]; the metric is irrelevant, since at the 
infinitesimal level all metrics are equivalent).

The following proposition provides some trivial but interesting examples.

3.8 Proposition. Let $M_{1}, M_{2}$ be smooth submanifolds of the unit sphere in $\mathbf{R}^{m_{1}} \times \mathbf{R}^{m_{2}}$. Let $p \in M_{2}$. Then the cone $C_{1}$ over $M_{1} \times p$ is area minimizing in the cone $C$ over $M_{1} \times M_{2}$.

Proof. The distance-nonincreasing retraction from $C$ to $C_{1}$, which carries $\left(t x_{1}, t x_{2}\right)$ to $\left(t x_{1}, t p\right)$, maps any competitor to $C_{1}$, which must therefore be area minimizing.

Example. Inside the 13-dimenisonal cone $C$ over $\mathbf{S}^{4} \times \mathbf{S}^{4} \times \mathbf{S}^{4}$ in $\mathbf{R}^{15}$, there are the following two 9-dimensional area-minimizing cones:

$C_{1}$ : the cone over $\mathbf{S}^{4} \times \mathbf{S}^{4} \times p$,

$C_{2}$ : the cone over $\mathbf{S}^{3} \times \mathbf{S}^{3} \times \mathbf{S}^{2}$.

The first cone $C_{1}$ is area minimizing by Proposition 3.8. The second cone $C_{2}$ is minimizing even in $\mathbf{R}^{15}$ [L, Thm. 5.1.1].

Other interesting examples are provided by Kerckhove and Lawlor [KL].

3.9 Unoriented surfaces. All of the results of this paper (and of [L]) apply as well in the larger context of unoriented surfaces or rectifiable currents modulo two (including orientable as well as nonorientable surfaces; see $[\mathrm{M} 1, \S 11.1])$.

3.10 Soap films. One may consider the context of soap films, technically $(\mathbf{M}, 0, d)$-minimal sets (see [M1, Sect. 11.3]). Which soap film hypercones, such as the cone over the regular simplex [LM], remain minimal under the metric 1(1) for some $\delta<1$ ? Perhaps a $k$-dimensional hypercone over a regular simplex remains minimal for some $\delta<1$ if and only if $k \geq 3$. It is easy to see that the 1-dimensional cone over the vertices of an equilateral triangle (the "Y" over the 2-simplex) does not even remain stable; indeed, has negative first variation. Computation after Simons [Sns, Lemma 6.1.6], generalized as in 3.1(6) above, shows that the 2-dimensional cone over the regular tetrahedron or 3-simplex is unstable. The singular points on the link in the sphere do not interfere (cf. [HMRR, Prop. 3.3]).

$k$-dimensional hypercones over cubes are known to be minimal if and only if $k \geq 3$ [B]. Brakke's calibrations [B], which prove them minimal for $k \geq 3$ with some leeway, imply that they remain minimal for some $\delta<1$. 


\section{References.}

[B] Kenneth A. Brakke, Minimal cones on hypercubes, J. Geom. Anal. 1 (1991) 329-338.

[CFG] Andrew Cotton, David Freeman, Andrei Gnepp, Ting Ng, John Spivack, Cara Yoder (Williams College NSF "SMALL" undergraduate research Geometry Groups 1998, 2000), The isoperimetric problem on singular surfaces, preprint (2000).

[F] Herbert Federer, Geometric Measure Theory, Springer-Verlag, 1969.

[G] Enrico Giusti, Minimal Surfaces and Functions of Bounded Variation, Birkäuser, Boston, 1984.

[HS] Robert Hardt and Leon Simon, Area minimizing hypersurfaces with isolated singularities, J. Reine Angew. Math. 362 (1985) 102-129.

[HMRR] Michael Hutchings, Frank Morgan, Manuel Ritoré, and Antonio Ros, Proof of the double bubble conjecture, Ann. Math. 155 (2002) 459-489.

[IT] Alexandr O. Ivanov and Alexei A. Tuzhilin, Minimal Networks: The Steiner Problem and its Generalizations, CRC Press, Boca Raton, 1994.

[KL] Michael Kerckhove and Gary Lawlor, A family of stratified areaminimizing cones, Duke Math. J. 96 (1999) 401-424.

[L] Gary R. Lawlor, A sufficient criterion for a cone to be areaminimizing, Mem. AMS, No. 446 (1991).

[LM] Gary R. Lawlor and Frank Morgan, Paired calibrations applied to soap films, immiscible fluids, and surfaces or networks minimizing other norms, Pacific J. Math. 166 (1994) 55-82.

[Ls] H. Blaine Lawson, The equivariant Plateau problem and interior regularity, Trans. AMS 173 (1972) 231-249.

[M1] Frank Morgan, Geometric Measure Theory: a Beginner's Guide, Academic Press, 3rd ed., 2000.

[M2] Frank Morgan, On the singular structure of two-dimensional area minimizing surfaces in $\mathbf{R}^{n}$, Math. Ann. 261 (1982) 101-110. 
[M3] Frank Morgan, Examples of unoriented area-minimizing surfaces, Trans. AMS 283 (1984) 225-237.

[Pet] Peter Petersen, Riemannian Geometry, Springer, 1998.

[SSY] R. Schoen, L. Simon, and S. T. Yau, Curvature estimates for minimal hypersurfaces, Acta Math. 134 (1975) 275-288.

[Se] Plinio Simoes, On a class of minimal cones in $\mathbf{R}^{n}$, Bull. AMS 80 (1974) 488-489.

[Sn] Leon Simon, Lectures on Geometric Measure Theory, Proc. Cent. Math. Anal. ANU 3 (1983).

[Sns] James Simons, Minimal varieties in riemannian manifolds, Ann. Math. 88 (1968) 62-105.

Department of Mathematics and Statistics

Williams College; Williamstown, MA 01267

USA

E-mail: Frank.Morgan@williams.edu

Received December 5, 2000. 